\title{
Vascularized composite allotransplantation combined with costimulation blockade induces mixed chimerism and reveals intrinsic tolerogenic potential
}

\author{
Byoung Chol Oh, ${ }^{1}$ Georg J. Furtmüller, ${ }^{1}$ Madeline L. Fryer, ${ }^{1}$ Yinan Guo, ${ }^{1,2}$ Franka Messner, ${ }^{1,3}$
} Johanna Krapf, ${ }^{4}$ Stefan Schneeberger, ${ }^{3}$ Damon S. Cooney, ${ }^{1}$ W.P. Andrew Lee, ${ }^{1}$ Giorgio Raimondi, and Gerald Brandacher ${ }^{1}$

'Department of Plastic and Reconstructive Surgery, Vascularized Composite Allotransplantation Laboratory, Johns Hopkins University School of Medicine, Baltimore, Maryland, USA. ${ }^{2}$ Department of Hand and Microsurgery, Xiangya Hospital, Central South University, Hunan, China. ${ }^{3}$ Department of Visceral, Transplant and Thoracic Surgery, and ${ }^{4}$ Department of Plastic and Reconstructive Surgery, Center of Operative Medicine, Medical University of Innsbruck, Innsbruck, Austria.

\begin{abstract}
Vascularized composite allotransplantation (VCA) has become a valid therapeutic option to restore form and function after devastating tissue loss. However, the need for high-dose multidrug immunosuppression to maintain allograft survival is still hampering more widespread application of VCA. In this study, we investigated the immunoregulatory potential of costimulation blockade (CoB; CTLA4-Ig and anti-CD154 mAb) combined with nonmyeoablative total body irradiation (TBI) to promote allograft survival of VCA in a fully MHC-mismatched mouse model of orthotopic hind limb transplantation. Compared with untreated controls (median survival time [MST] 8 days) and CTLA4-Ig treatment alone (MST 17 days), CoB treatment increased graft survival (MST 82 days), and the addition of nonmyeloablative TBI led to indefinite graft survival (MST > 210 days). Our analysis suggests that VCA-derived BM induced mixed chimerism in animals treated with CoB and TBI + CoB, promoting gradual deletion of alloreactive $T$ cells as the underlying mechanism of long-term allograft survival. Acceptance of donor-matched secondary skin grafts, decreased ex vivo $\mathrm{T}$ cell responsiveness, and increased graft-infiltrating Tregs further indicated donor-specific tolerance induced by TBI + CoB. In summary, our data suggest that vascularized BM-containing VCAs are immunologically favorable grafts promoting chimerism induction and long-term allograft survival in the context of CoB.
\end{abstract}

Authorship note: BCO and GJF contributed equally to this work.

Conflict of interest: The authors have declared that no conflict of interest exists.

Copyright: (c) 2020, American Society for Clinical Investigation.

Submitted: March 4, 2019

Accepted: March 4, 2020

Published: March 17, 2020.

Reference information: JCI Insight. 2020;5(7):e128560.

https://doi.org/10.1172/jci.

insight.128560.

\section{Introduction}

Vascularized composite allotransplantation (VCA) has become a clinical reality, with more than 130 hand/ forearm/arm transplants and 40 face transplants performed worldwide with highly encouraging immunological and functional outcomes (1). Despite these successes, wider utilization of VCA is hindered by the need for long-term, high-dose immunosuppression. Thus, there is an imminent need to develop novel strategies to maintain graft survival while reducing adverse effects and toxicities of conventional immunosuppression. The use of costimulation blockade $(\mathrm{CoB})$ via biologic agents such as the recombinant Cytotoxic T-Lymphocyte-associated antigen-4 Ig (CTLA4-Ig) - e.g., abatacept and belatacept, which block T-cell costimulation — have been developed to overcome these limitations.

CTLA4-Ig has shown considerable efficacy in preventing rejection of solid organ transplants in preclinical models and clinical trials (2-6). However, CTLA4-Ig alone cannot induce long-term graft survival or tolerance in any stringent murine transplant model (e.g., Balb/C into C57BL/6) (7, 8). As shown in various small and large animal models of solid organ and skin transplantation, the addition of antiCD154 mAb or donor-specific transfusion (DST) to CTLA4-Ig treatment prevented acute and chronic rejection and led to long-term allograft survival and tolerance $(7,9-12)$. In particular, the combination 
of $\mathrm{CoB}$ with $\mathrm{BM}$ transplantation (BMT) has been an effective means to establish mixed chimerism and induce tolerance in rodent models $(13,14)$. This finding carries increased relevance in VCA, as certain composite tissue allografts comprise vascularized BM (vBM), a graft component that could confer the inherent ability to promote tolerance by inducing mixed chimerism when combined with CoB treatment - a feature distinctly not present in solid organ transplantation. Therefore, the focus of this study was to test the efficacy of $\mathrm{CoB}$ on allograft survival and the investigation of mechanisms underlying the promotion of immunological tolerance in a murine model of transplantation of vBM-containing allografts.

Here, we were able to report on means to induce long-term allograft survival without the requirement of conventional maintenance immunosuppressive therapy in the context of $\mathrm{CoB}$ and VCA. In addition, we showed unique insight into chimerism-based long-term VCA survival promoted by a vBM-containing allograft and $\mathrm{CoB}$ in a fully MHC-mismatched mouse model.

\section{Results}

CoB promotes VCA survival. To test the effect of $\mathrm{CoB}$ on VCA survival, we used our recently reported murine orthotopic hind limb transplant model (15) performed across a full MHC barrier: from Balb/C $\left(\mathrm{H}-2^{\mathrm{d}}\right.$ ) into C57BL/6 (H-2 $)$ (Figure 1, A-C). Treatment with CTLA4-Ig alone showed prolonged VCA survival (median survival time [MST] 17 days) as compared with untreated controls (MST 8 days; $P<$ 0.01). Combined CoB treatment with CTLA4-Ig + MR1 (CoB) significantly increased VCA survival (MST 82 days; $P<0.01$ ) compared with untreated controls and animals receiving CTLA4-Ig only. The addition of $250 \mathrm{cGy}$ of nonmyeloablative total body irradiation (TBI) led to indefinite allograft survival without clinical rejection episodes (MST $>210$ days; $P<0.01$ ) (Figure 1D). Controls that only received nonmyeloablative TBI showed a MST of 13.3 days (Supplemental Figure 1; supplemental material available online with this article; https://doi.org/10.1172/jci.insight.128560DS1).

Histologic analysis correlated well with clinical graft survival. In untreated controls, signs of rejection - including epidermolysis and massive mononuclear cell infiltration - were present at postoperative day (POD) 7 (Figure 2, A and B). CTLA4-Ig only-treated recipients showed Grade 3 rejection with a diffuse cellular infiltrate on $\mathrm{H} \& \mathrm{E}$ staining (Figure 2C). However, around $50 \%$ of recipients treated with $\mathrm{CoB}$ showed only mild infiltration in the dermis, without clinical evidence of skin rejection episodes at POD 70 (Figure 2, D and E). Recipients receiving TBI + CoB showed neither clinical rejection signs nor cellular infiltration (Figure 2, F-H). In both groups, no donor specific antibodies (DSA) formation was detectable (mean fluorescence intensity $[\mathrm{MFI}] \pm \mathrm{SEM}$ ) at POD 70 (CTLA4-Ig + MR1, $1049 \pm$ 118.5, vs. TBI + CTLA4-Ig + MR1, $1028 \pm 50.13$; nontransplanted control, $1051 \pm 90.47$, vs. positive control [serum collected 70 days after allo-skin rejection], $11041 \pm 1133)$. These results suggest that CoB promotes immune-modulatory mechanisms intrinsic to the VCA model - in particular, when considering the limited efficacy of $\mathrm{CoB}$ on the survival of full-thickness skin transplants as previously reported (16).

CoB promotes multilineage donor chimerism. Donor chimerism emerged as a key element of successful tolerance induction protocols in solid organ transplantation (17). Therefore, we tested the ability of a vBM containing allograft to induce donor-specific chimerism. We analyzed the presence of donor-derived PBMCs among all PBMCs at multiple time points after transplantation using flow cytometry. Microchimerism was detectable on POD 7 (percentage of $\mathrm{CD}_{11} \mathrm{~b}^{+} \mathrm{H}-2 \mathrm{~d}^{+}[$mean $\pm \mathrm{SEM}] ; 0.752 \% \pm 0.386 \%$ ) but disappeared by POD 13 (percentage of $\mathrm{CD} 11 \mathrm{~b}^{+} \mathrm{H}-2 \mathrm{~d}^{+} ; 0.016 \% \pm 0.006 \%$ ) in untreated controls. In CTLA4-Ig-treated recipients, mixed chimerism was detectable up to POD 13 (percentage of CD11b $\mathrm{H}_{-}$ $2 \mathrm{~d}^{+} ; 0.6427 \% \pm 1.347 \%$ ) and then disappeared as the VCA was rejected (Figure 3C). Similar patterns were detected for donor-derived $\left(\mathrm{H}-2 \mathrm{~d}^{+}\right.$-derived) $\mathrm{CD}^{+} \mathrm{T}$ cells and $\mathrm{B} 22 \mathrm{0}^{+} \mathrm{B}$ cells in both groups (Figure $3, \mathrm{~A}$ and $\mathrm{B}$ ). In recipients treated with the $\mathrm{CoB}$ regimen, the percentage of donor-derived $\mathrm{B} 220^{+}$cells and $\mathrm{CD} 11 \mathrm{~b}^{+}$cells present in the blood remained stable in the range of $4 \%-8 \%$ until POD 50 and then gradually decreased (Figure 3, B and C). In mice treated with $\mathrm{TBI}+\mathrm{CoB}$, stable mixed chimerism of donor-derived $\mathrm{CD}^{+}, \mathrm{B}_{22} 20^{+}$, and $\mathrm{CD} 11 \mathrm{~b}^{+}$donor cells, accounting for up to $16 \%$ of circulating PBMCs, was observed indefinitely (Figure 3, A-C, and Supplemental Figure 2). This indicated that the addition of nonmyeloablative TBI further increased and stabilized the level of chimerism throughout animal survival and correlated with graft survival.

Effect of $C o B$ on $T$ cell reactivity. To assess if the extended survival correlated with a systemic alteration of $\mathrm{T}$ lymphocyte alloreactivity, antidonor $\mathrm{T}$ cell proliferation was tested using CFSE-mixed lymphocyte reactions (CFSE-MLR). Both $\mathrm{CoB}-$ and $\mathrm{TBI}+\mathrm{CoB}$-treated groups exhibited significantly reduced $\mathrm{CD}^{+}$ 

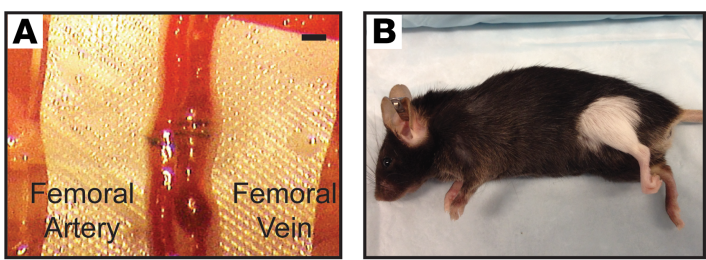

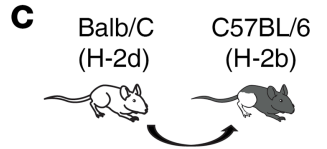

Day of transplant
Survival mixed chimerism in vitro assays
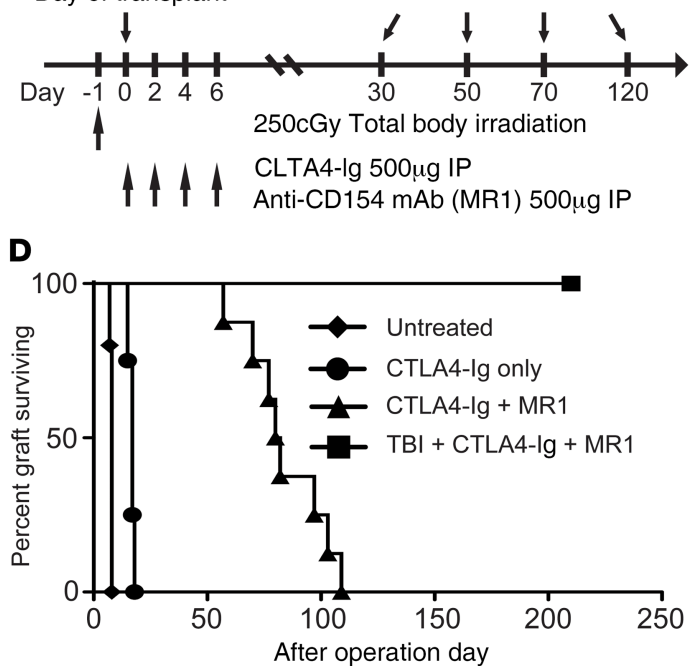

Figure 1. Experimental design, treatment regimen, and vascularized composite allograft survival. (A and B) An orthotopic hind limb transplantation model based on the nonsuture cuff technique was used to allow for clinical allograft survival monitoring. (A) Representative image of microvascular anastomosis using polyethylene cuff tubes. (B) Long-term survivor on POD 120. (C) Schematic representation of the transplant strategy implemented and the different treatment strategies investigated. These included combination of total body irradiation, CTLA4-Ig, and anti-CD154 mAb (MR1). mixed chimerism analyses combined with other in vitro assays were performed as outlined. (D) Hind limb allograft survival was prolonged with CTLA4-Ig and MR1 without total body irradiation treatment (MST 82 days, $n=8$; MST >210 days, $n=6 ; P=0.0008$ ), while untreated and CTLA4-Ig only-treated recipients showed acute rejection at MST of 8 days $(n=5)$ and 15 days $(n=4)$. $P$ values were calculated by log-rank test $(D)$.

and $\mathrm{CD}^{+} \mathrm{T}$ cell proliferation against donor-derived $\mathrm{DC}$ compared with proliferation of the same cell populations from naive mice. However, the response to third-party DC (Figure 4) was not affected by either treatment, suggesting a donor-specific alteration of alloreactivity.

In addition, allogeneic effector and memory $\mathrm{T}$ cell responses were investigated ex vivo. Before treatment and at specific time points after transplant (Figure 5, A and B), lymphocytes were purified and briefly restimulated in vitro to assess the presence of effector/memory function via intracellular staining for IFN- $\gamma$. Compared with untreated controls (POD 7), $\mathrm{CoB}$ and TBI + $\mathrm{CoB}$ treatments inhibited the accumulation of IFN- $\gamma$-producing CD4 ${ }^{+}$T cells $(6.04 \% \pm 0.32 \%$ vs. $1.53 \% \pm 0.15 \%$ and $1.17 \% \pm 0.20 \%$, respectively; $P<0.01)$ at POD 50 (Figure $5 \mathrm{~A})$. By POD 70, increased IFN- $\gamma$ production was detected in recipients treated with CoB compared with TBI $+\mathrm{CoB}$ (percentage of $\mathrm{CD} 4^{+} \mathrm{IFN}-\gamma^{+} \mathrm{T}$ cells; $4.34 \% \pm 0.58 \%$ vs. $1.75 \% \pm 0.37 \%$, respectively; $P<0.05$ ) (Figure $5 \mathrm{~A})$. In $\mathrm{CD}^{+} \mathrm{T}$ cells, IFN- $\gamma$ production was significantly higher in animals treated with $\mathrm{TBI}+\mathrm{CoB}$ compared with CoB at POD 30 (percentage of $\mathrm{CD}^{+} \mathrm{IFN}-\gamma^{+} \mathrm{T}$ cells; $30.23 \% \pm 2.325 \%$ vs. $16.13 \% \pm 2.894 \%$, respectively; $P<0.01$ ) and POD 50 (percentage of CD ${ }^{+} \mathrm{IFN}-\gamma^{+}$T cells; $17.28 \% \pm 2.909 \%$ vs. $11.00 \% \pm 2.336 \%$, respectively; $P<0.01$ ) (Figure 5B). In addition, the CoB group showed a significant increase of IFN- $\gamma$ production between POD 50 and POD 70 (percentage of $\mathrm{CD}^{+} \mathrm{IFN}^{-} \gamma^{+} \mathrm{T}$ cells; $11.00 \% \pm 2.336 \%$ vs. $19.58 \% \pm 3.325 \%$, respectively; $P<0.05$ ) that correlated with the pattern seen in $\mathrm{CD}^{+} \mathrm{T}$ cells (Figure 5, A and B). No differences, however, were seen for other intracellular cytokines such as IL-2, IL-10, and IL-17 (Supplemental Figure 3). Collectively, these data indicate that $\mathrm{T}$ cell reactivity was suppressed (in an antigen-specific fashion) in VCA recipients treated with CoB. Graft survival inversely correlated with $\mathrm{T}$ cell recovery in CoB only-treated animals. 


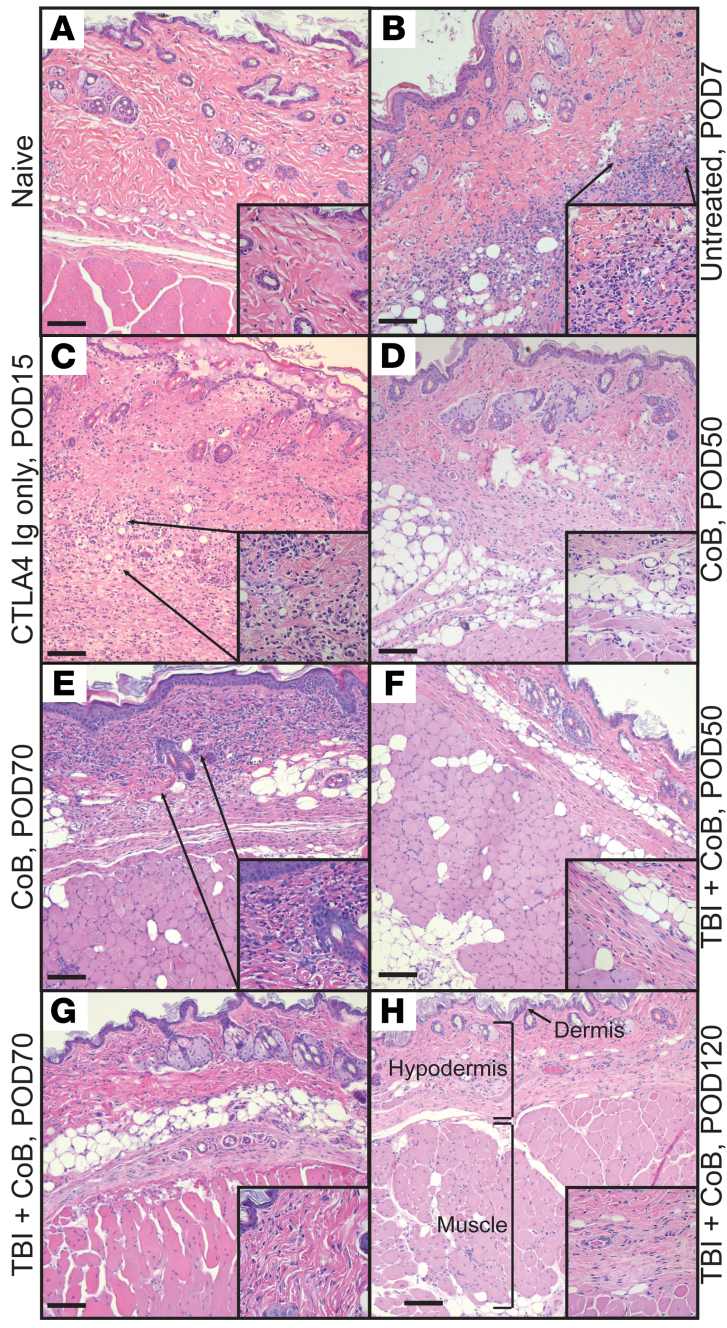

Figure 2. Representative H\&E staining. (A and B) Soft tissues of the hind limb of a naive control animal are compared with transplanted hind limb allografts in untreated control animals on POD7 and compared with animals treated with various treatment combinations. (C) CTLA4-Ig only (POD 15). (D) CoB (POD 50). (E) CoB (POD 70). (F) TBI + CoB (POD 50). (G) TBI + COB (POD 70). (H) TBI + COB (POD 120). Scale bar: $100 \mu \mathrm{m}$. Original magnification, $\times 100$. Inset magnification, $\times 400$ with arrows to indicate cellular infiltration.

Graft infiltration of $\mathrm{FoxP}^{+} \mathrm{T}$ cells. Tregs play a critical role in maintaining allograft survival in organ transplantation but are not sufficient on their own to induce skin graft tolerance across full MHC barriers in rodent models $(18,19)$. To further study the critical role of Tregs, we examined the composition of the cellular infiltrate in the VCA after $\mathrm{CoB}$ treatment, with particular focus on $\mathrm{CD} 4^{+} \mathrm{T}$ effector cells and $\mathrm{CD} 4^{+}$ Foxp3 ${ }^{+}$Tregs. The proportion of cells expressing Foxp3 was reduced in the untreated control animals compared with nontransplanted naive mouse hind limbs (POD 7, $10.02 \pm 1.05$ vs. $14.31 \pm 0.77$, respectively; $P$ $<0.01)$. At POD 30, fewer infiltrating $\mathrm{CD} 4^{+} \mathrm{Foxp}^{+} \mathrm{T}$ cells were found in CoB-treated allografts compared with TBI + CoB-treated allografts $(11.11 \pm 1.74$ vs. $40.13 \pm 5.31$, respectively; $P<0.01)$. On POD 50, higher numbers of $\mathrm{CD}^{+} \mathrm{Foxp}^{+} \mathrm{T}$ cells were found compared with early time points in both treatment groups and naive and untreated controls $(34.39 \pm 4.50$ in CoB-treated and $38.03 \pm 3.56$ in TBI + CoB-treated vs. naive and untreated control; $P<0.05)$. Starting on POD 70, a decline of $\mathrm{CD}^{+} \mathrm{FoxP}^{+} \mathrm{T}$ cell frequency was observed in animals treated with $\mathrm{CoB}(29.95 \pm 1.70)$ and $\mathrm{TBI}+\mathrm{CoB}(17.55 \pm 3.99)$. Interestingly, animals in latter group had fewer $\mathrm{CD}^{+} \mathrm{FoxP}^{+}$cells compared with CoB-treated recipients $(17.55 \pm 3.40$ vs. 29.95 \pm 1.70 , respectively; $P<0.05$ ). To study the functionality of Tregs, we assess the suppressive activity of $\mathrm{CD}^{+}{ }^{+} \mathrm{Foxp}^{+}$Tregs. Tregs isolated from recipients treated with TBI $+\mathrm{CoB}$ showed stronger suppression compared with $\mathrm{CoB} 70$ days after transplant. Tregs isolated from the $\mathrm{CoB}$ group showed no differences of suppressive activity compared with natural Tregs isolated from WT C57BL/6 mice (Supplemental Figure 4). To further study the origin of these Tregs, donor haplotype expression in the Foxp $3^{+}$Tregs was examined in spleen. Frequencies of Tregs expressing donor MHC class I haplotype were significantly higher in the group of TBI $+\mathrm{CoB}$ compared with $\mathrm{CoB}(48.98 \pm 15.38$ vs. $0.5775 \pm 0.337$, respectively; $P<0.05)$, while the proportion of cells expressing Foxp3 in the spleen was similar between both groups on POD70 $(9.978 \pm 1.638$ vs. $11.64 \pm 0.8664$, respectively; $P>0.05)$ (Supplemental Figure 5$)$. However, after in vivo 
A
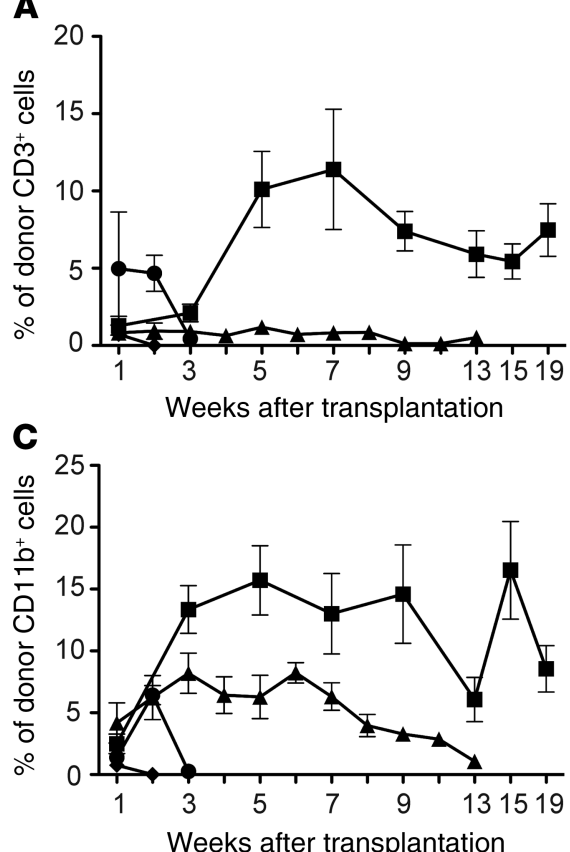

B

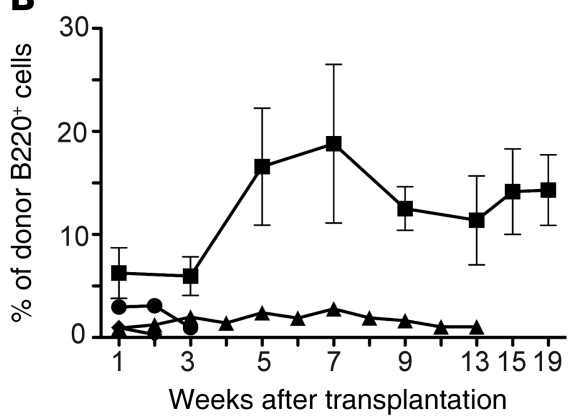

Weeks after transplantation

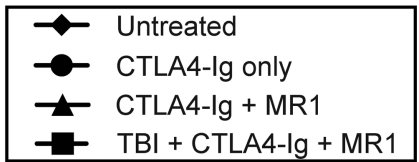

Figure 3. Multilineage PBMC donor chimerism. Donor-specific multilineage mixed chimerism was detected in animals treated with $\mathrm{CoB}$, indicating viable donor hematopoiesis derived from the graft inherent donor BM compartment. The addition of TBI further increased mixed chimerism levels ( $n=3-7 /$ time point). (A) Donor-derived CD3 ${ }^{+} \mathrm{T}$ cells. (B) Donor-derived B220+ B cells. (C) Donor-derived CD11b+ myeloid cells. The percentage of expression of donor MHC class I for each lineage circulating in host peripheral blood was calculated. Data are representative of at least 3 mice per group from 2-3 independent experiments.

depletion of Tregs using PC61, graft survival - as well as induction of mixed chimerism - was not affected (Supplemental Figure 6). The superior suppressive activity of Tregs isolated from animals treated with TBI + CoB suggest an important protective role for Tregs in maintaining allograft survival. However, the differences in the dynamics between $\mathrm{COB}$ and $\mathrm{TBI}+\mathrm{CoB}$ treatments underscored the importance of central mechanisms of regulation in the establishment of immune tolerance toward a VCA.

$T B I+C o B$ induces robust tolerance to donor antigens via central deletion. To examine the effect of $C o B$ and chimerism on thymic central deletion of donor-reactive $\mathrm{T}$ cells, we analyzed the occurrence of certain donor-reactive V $\beta \mathrm{T}$ cell receptor (TCR) subunits in PBMCs as previously described by Wekerle et al. (14). Briefly, developing thymocytes whose $\mathrm{T}$ cell receptors contain $\mathrm{V} \beta 11$ or $\mathrm{V} \beta 5.1 / 2$, which bind to these superantigens, are deleted in I-E $\mathrm{E}^{+} \mathrm{Balb} / \mathrm{C}$ mice - but not in B6 mice because they do not express I-E (20). In mice receiving either $\mathrm{CoB}$ or $\mathrm{TBI}+\mathrm{CoB}$, reduced frequencies of $\mathrm{V} \beta 5^{+}$and $\mathrm{V} \beta 11^{+} \mathrm{CD} 4^{+} \mathrm{T}$ cells were observed by POD 50, indicating ongoing deletion of donor-reactive $\mathrm{T}$ cells. In recipients treated with $\mathrm{TBI}+\mathrm{CoB}$, deletion of these alloreactive ${\mathrm{V} \beta 5^{+}}^{+}$and $\mathrm{V} \beta 11^{+} \mathrm{T}$ cells was sustained throughout the follow-up period (>120 days), while remaining only partial in animals treated with $\mathrm{CoB}$ alone (Figure 6). Extended graft survival correlated with higher levels of chimerism and V $\beta$ TCR deletion at a later stage for this group (Figure 6, A and B). This delayed or partial deletion was particularly observed in animals in which chimerism was lost (MST 82 days; compare with Figure 3). Frequencies of control V 88 TCR-expressing $\mathrm{CD}^{+} \mathrm{T}$ cells, which do not recognize donor-derived superantigens, were not reduced at any time point, representing normally occurring thymic selection (21) (Figure 6C). To further investigate the role of donor $\mathrm{BM}$ in tolerance induction, thymic tissue was collected from long-term-surviving animals. The frequency of $\mathrm{CD} 11 \mathrm{c}^{+} \mathrm{DCs}$ expressing donor MHC class I haplotype $\left(\mathrm{H}-2 \mathrm{~K}^{\mathrm{d}}\right)$ was significantly higher in recipients treated with $\mathrm{TBI}+\mathrm{CoB}$ compared with $\mathrm{CoB}$ alone $(15.64 \pm 2.647$ vs. $2.745 \pm 0.9207$, respectively; $P<$ 0.01 ) (Supplemental Figure 7). These results suggest thymic engraftment of donor-derived cells in VCA recipients treated with $\mathrm{TBI}+\mathrm{CoB}$, allowing for thymic deletion of donor-reactive $\mathrm{T}$ cells.

Graft acceptance of secondary skin grafts in long-term survivors. Donor skin graft survival is commonly regarded as a stringent test for assessing transplantation tolerance. We therefore subjected long-term survivors (>POD 120) 

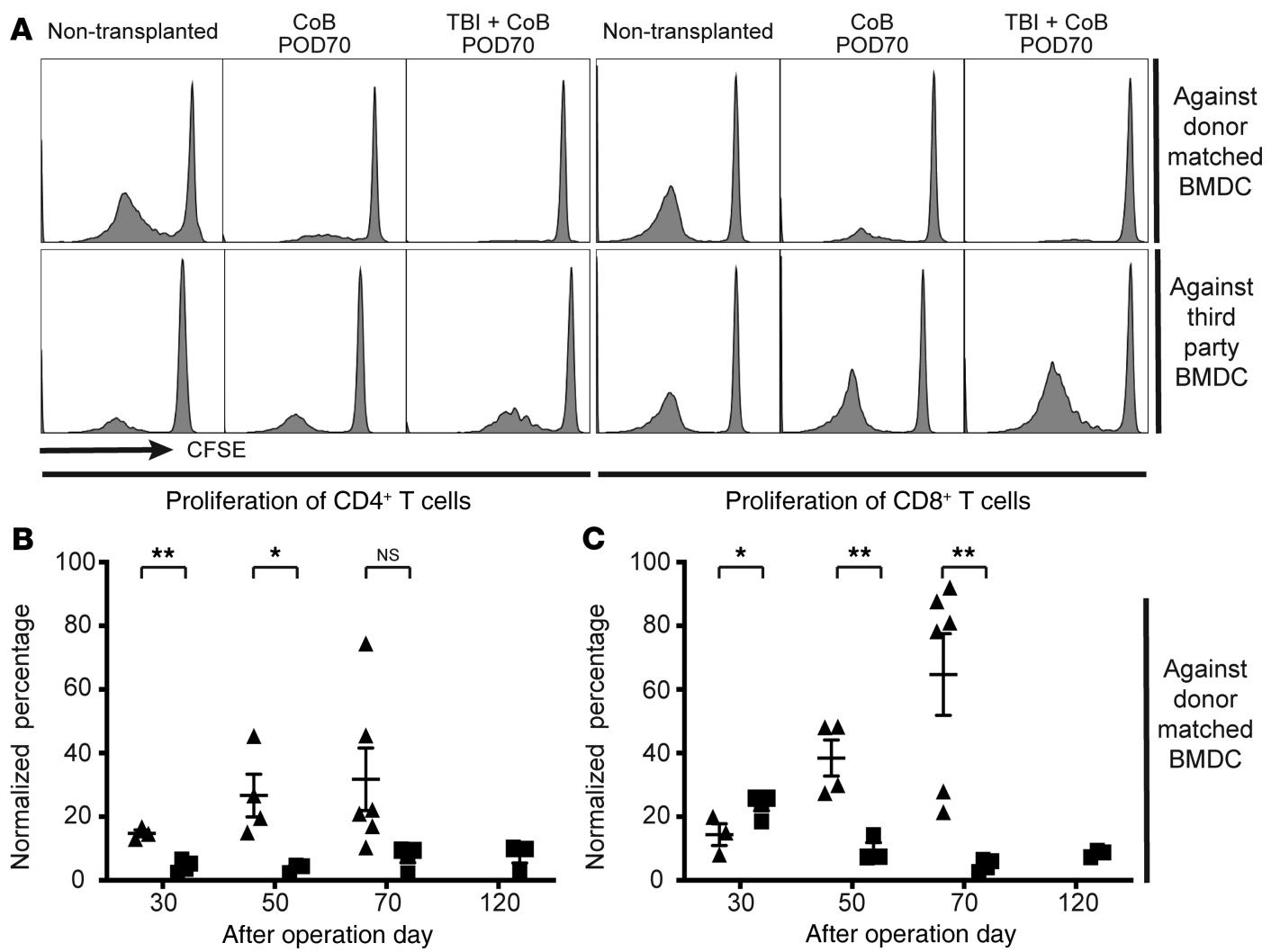

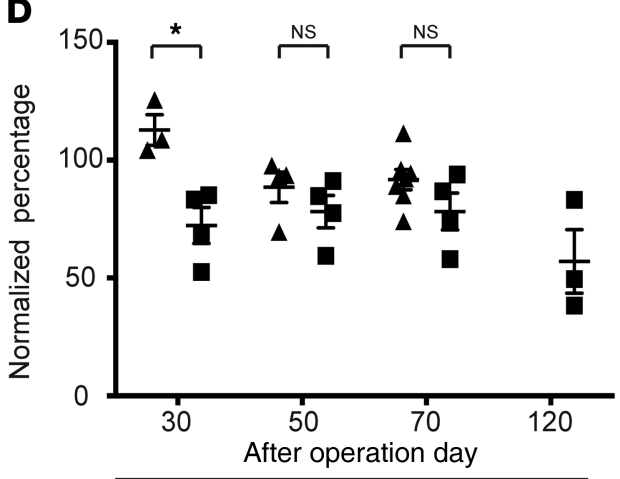

Proliferation of $\mathrm{CD}^{+} \mathrm{T}$ cells
$\mathbf{E}$

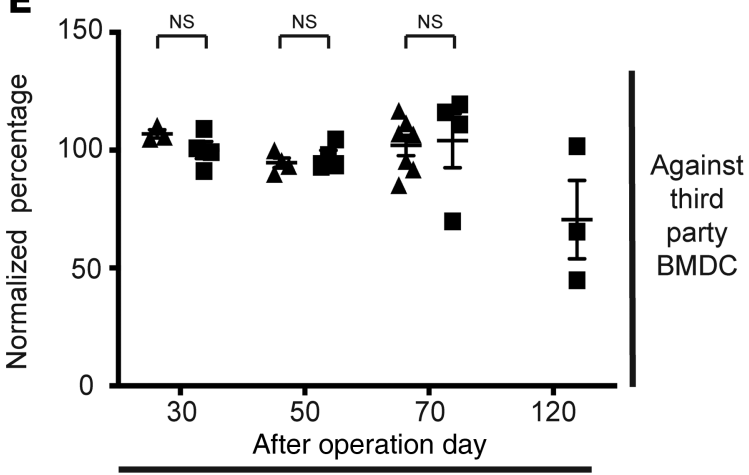

Proliferation of $\mathrm{CD}^{+} \mathrm{T}$ cells

$$
\begin{array}{ll}
\boldsymbol{\Delta} & \text { CTLA4-Ig + MR1 } \\
& \text { TBI + CTLA4-Ig + MR1 }
\end{array}
$$

Figure 4. Mixed lymphocyte reactions to assess $\mathrm{T}$ cell alloreactivity. T cells cocultures were performed at various time points after transplantation. (A) Representative dilution of CFSE measured by flow cytometry (B) Decreased CD4 ${ }^{+} T$ cell proliferation against donor-derived DC was observed in the group treated with $\mathrm{TBI}+\mathrm{CoB}$ compared with the CoB treatment alone. (C) In CD8 ${ }^{+} \mathrm{T}$ cells, CoB-treated recipients showed significantly increased proliferation against donor-derived $D C$ at POD 50 and POD 70 compared with mice treated with TBI + CoB. TBI + CoB recipients displayed a decreasing trend of $T$ cell proliferation against donor-derived DC from POD 30 to POD 70. (D and E) CD4+ and CD8 ${ }^{+}$proliferation was maintained against third-party-derived DC in both treatment groups ( $n=3-8$ /time point and group; mean $\pm \mathrm{SD},{ }^{*} P<0.05,{ }^{* *} P<0.01 ; P$ values were calculated by 2 -tailed $t$ test).

to a secondary challenge with donor-matched and third-party full-thickness skin grafts (Figure 7A). Long-term survivors treated with TBI + CoB accepted donor-matched skin for the entire 80-day follow-up period, while maintaining the ability to reject third-party skin grafts (Figure 7B). To further test the robustness of the observed tolerance phenomenon, we injected naive, recipient-matched $\mathrm{T}$ cells into long-term VCA survivors (Figure 7A) accepting the primary VCA and a secondary skin graft for 210 and 90 days, respectively (the TBI + CoB group). Animals rejected both grafts after 17-22 days (MST 19.33 days) (Figure 7C). These results demonstrate robust donor-specific tolerance in vivo; however, peripheral regulatory mechanisms underlying this phenomenon remain insufficient to prevent allograft rejection by naive recipient-type $\mathrm{T}$ cells. 

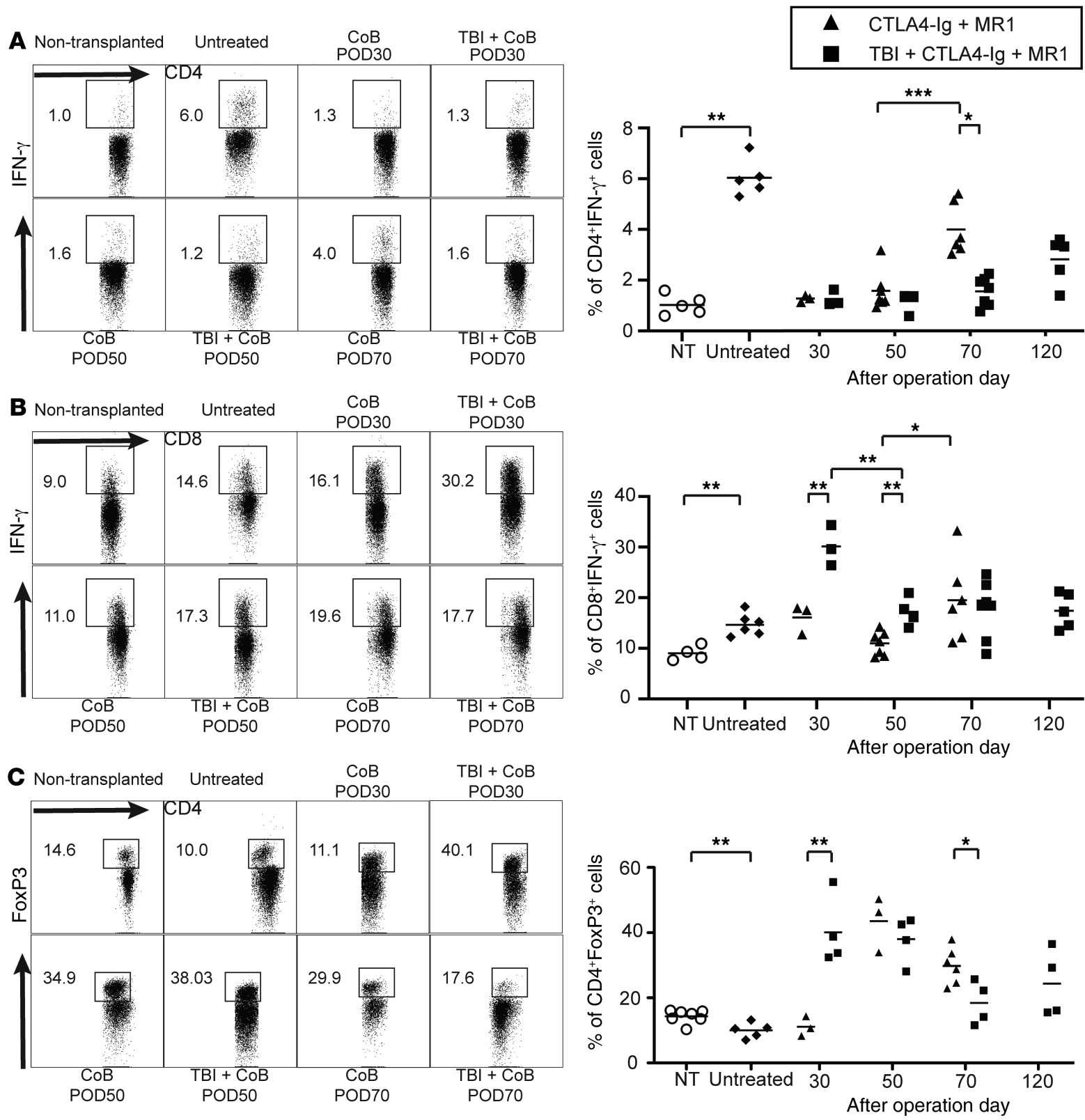

Figure 5. Effect of costimulation blockade on T cell reactivity. (A) Representative gating strategy for the detection of IFN- $\gamma$ production. The production of IFN- $\gamma$ in recipient lymphocytes was measured to determine CD4+ T cell reactivity. IFN- $\gamma$ production was significantly increased in CoB-treated recipients at POD 70 compared with TBI + CoB-treated recipients. NT, nontransplanted control. (B) The production of IFN- $\gamma$ in recipient lymphocytes was measured to determine CD8 T cell reactivity. IFN- $\gamma$ production was significantly increased in CoB-treated recipients at POD 70 compared with POD 50. (C) Graft infiltration by CD4 ${ }^{+}$FoxP3 ${ }^{+} T$ cells was compared across treatment groups and time points in long-term survivors. On POD 30, the proportion of infiltrating CD4 ${ }^{+} \mathrm{T}$ cells positive for FoxP3 ${ }^{+}$ was increased in $\mathrm{TBI}+\mathrm{CoB}$-treated recipients compared with $\mathrm{CoB}$-treated recipients. $\mathrm{CD} 4^{+} \mathrm{FoxP}^{+} \mathrm{T}$ cell infiltration increased in the group treated with $\mathrm{CoB}$ at

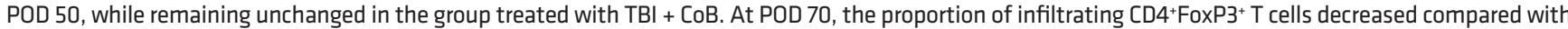
POD 50 in TBI + CoB-treated recipients ( $n=3-8$ /time point and group; bar represents mean; ${ }^{*} P<0.05,{ }^{* *} P<0.01$; $P$ values were calculated by 2 -tailed $t$ test).

\section{Discussion}

Current research in VCA focuses on determining significant differences in immunologic features between solid organ transplants and vascularized composite allografts. In particular, a detailed understanding of the alloimmune response toward various graft and tissue types may enable the development of novel strategies for immunomodulation and tolerance induction specifically tailored to VCA to tilt the risk benefit ratio in favor of these non-life saving types of transplants (22). In this study, we aimed to investigate a potentially novel strategy to prevent VCA rejection by using CoB by means of CTLA4-Ig and MR-1 combined with nonmyeloablative induction therapy (Figure 1, B and C). 

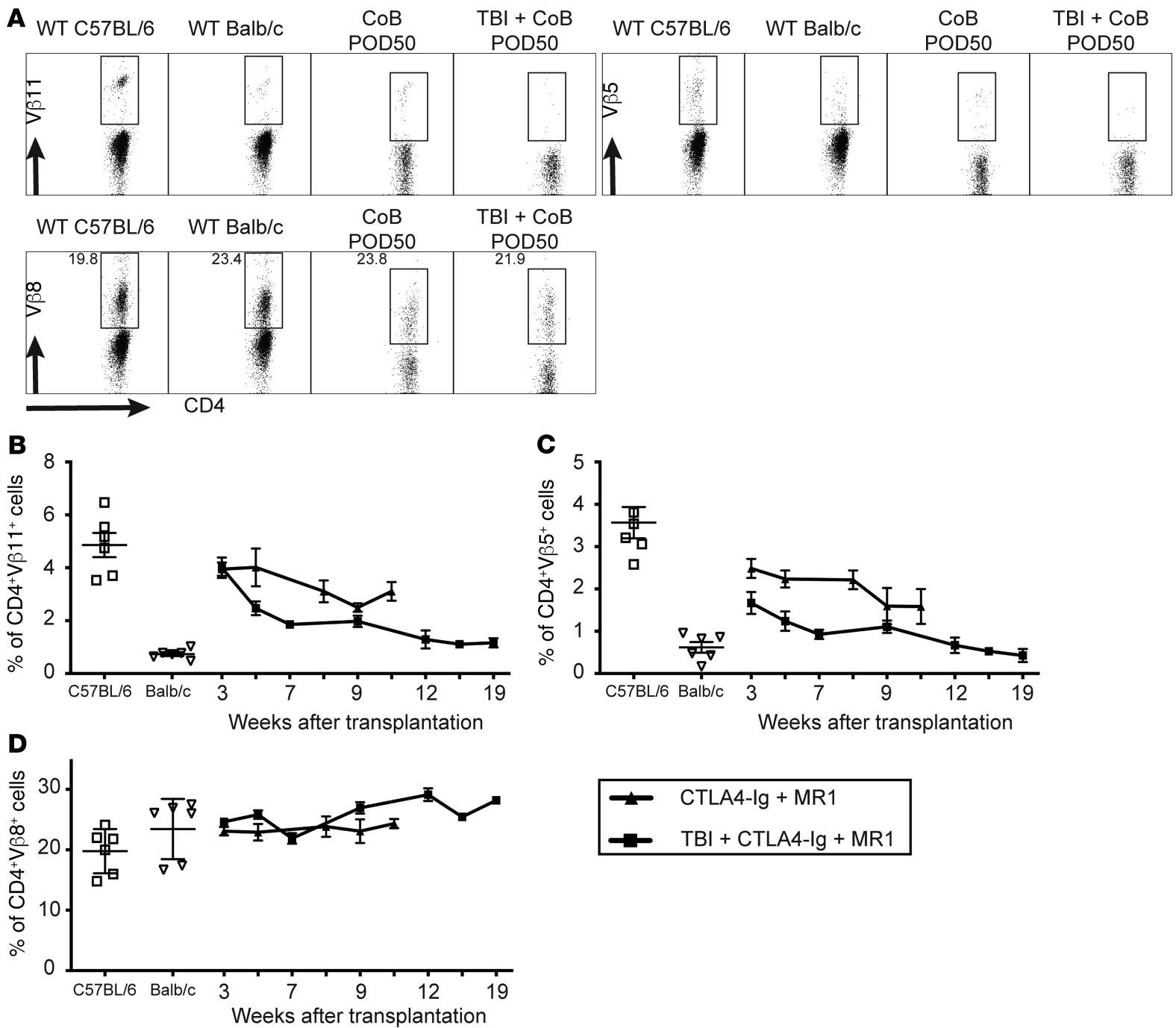

Figure 6. Central deletion of donor reactive T cells. V $\beta$ TCR markers were used to detect donor-reactive T cells from PBMCs. Deletion of V 35 and 11 TCRs represents central deletion based on thymic engraftment of donor cells as long as chimerism persists ( $n=3-7 /$ time point). White and black bars represent expression from naive $\mathrm{C57BL} / 6$ and Balb/C controls. (A) Representative gating strategy for detection of V $\beta$ TCR expressions. (B) TBI + CoB-treated animals showed deletion of donor-reactive $C D 4^{+} V \beta 11^{+} T$ cells, while CoB-treated recipients showed partial deletion. (C) $C D 4^{+} V \beta 5.1 / 2^{+} T$ cells were deleted in recipients treated with TBI + CoB compared with partial deletion in CoB-treated animals. (D) Positive control V $\beta 8^{+}$TCR expressing CD4 ${ }^{+}$T cells were not deleted in either group.

The application of CoB-based treatment protocols has allowed for significant progress toward critical goals in transplantation, including the need to reduce overall toxicity of high-intensity induction regimens and long-term immunosuppression - in particular, in BMT $(23,24)$. Furthermore, in addition to its potential application as a primary immunosuppressant allowing minimization or withdrawal of calcineurin inhibitors, rodent models employing CTLA4-Ig suggest that belatacept may have beneficial and critical effects in tolerance-inducing regimens. In a previous study, we showed that CoB-based induction therapy, followed by short-term maintenance with conventional immunosuppression, facilitates vascularized osteomyocutaneous allograft survival in rodents (25). However, CTLA4-Ig alone remains ineffective in prolongation of graft survival and tolerance induction without the addition of either donor splenocyte transfusion (DST) or anti-CD154 antibodies (MR1) (7, 11, 26, 27). There is unequivocal evidence demonstrating that CTLA4-Ig alone is not capable of inducing tolerance in either stringent rodent models $(11,27)$ or in nonhuman primate solid organ transplantation $(28,29)$. Even in less-stringent murine heart allograft models, DST or anti-CD40L have to be combined with CTLA4-Ig treatment in order to achieve indefinite graft survival, which is still associated with signs of chronic rejection (30). Thus, it seems clear that CTLA4-Ig must be combined with other strategies if tolerance is the ultimate goal. Though very effective in small and large preclinical animal models, the combination of CTLA4-Ig 
A Balb/C C57BL/6

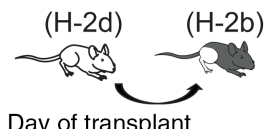

Day of transplant

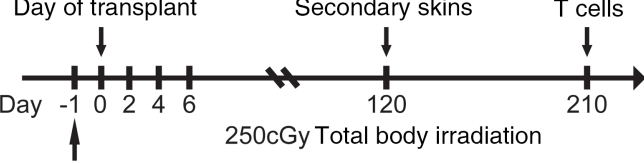

1411 CLTAA-Ig 500ug IP Anti-CD154 mAb (MR1) 500ug IP

B
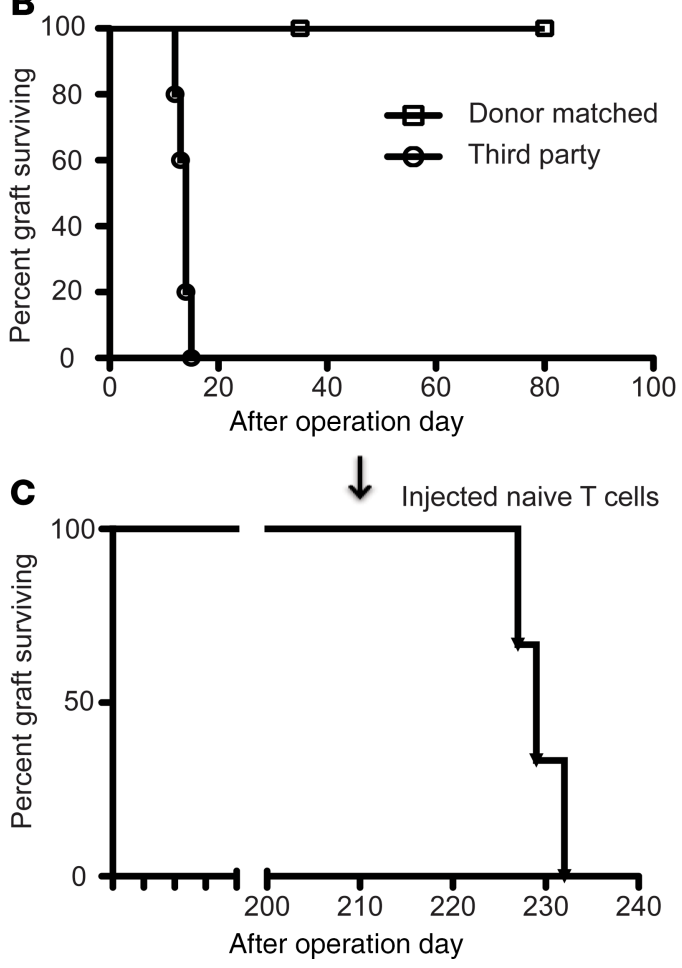

Figure 7. Assessment of donor-specific tolerance. (A) Recipients were treated with 250 cGy TBI + CTLA4-Ig + MR1. Long-term survivors were monitored for rejection following subsequent secondary skin grafts and naive T cell injection. (B) Secondary full-thickness skin transplantation was performed in long-term survivors ( $\mathrm{TBI}+\mathrm{CoB}$-treated, >POD 120) with donor-matched (Balb/C) and third-party (FVB/N) allografts. Long-term survivors accepted second donor-matched skin but rejected third-party-derived skin in MST 14 days $(n=5)$. (C) Eighty days after secondary skin transplantation, long-term survivors were injected with $20 \times 10^{6}$ naive C57BL/6 T cells. Recipients rejected original hind limb graft and secondary skin graft between 17 and 22 days after injection $(n=3)$. Statistical differences were calculated by log-rank test.

and anti-CD40L mAb is due the high incidence of thromboembolic events of the latter not yet available in human clinical trials. Thus, administration of donor BM cell infusion/transplantation in combination with CTLA4-Ig appears to be the most promising approach in this regard, as indicated by various small and large animal solid organ transplant models. This is particularly attractive for VCA, as - in many circumstances - the transplanted tissues include a vascularized BM graft component that serves as a BM transplant itself $(31,32)$. Thus, when combined with donor BM infusion or with grafts containing a vascularized BM component, VCA represent an exciting opportunity to unleash the tolerogenic potential of costimulatory blockade. Previous studies indicated that the combined use of CoB and BM infusion allowed for a reduction of the intensity of the preconditioning regimen, as well as the replacement of irradiation with administration of busulfan, to establish therapeutic mixed chimerism (33). Despite the aforementioned limitations, CD40/CD154 interaction plays a critical role in the regulation of immune responses during allograft rejection; therefore, the development of alternative and clinically applicable regimens remains very attractive. Several candidate agents are currently under investigation, including ICOS, as possible agents that could be used synergistically with CTLA4-Ig $(34,35)$.

In our current model, $\mathrm{CoB}$ alone also did not induce tolerance or sustain any significant level of chimerism and merely prolonged graft survival. Induction of stable donor-specific mixed chimerism and indefinite allograft survival could only be achieved when TBI was added to the regimen and combined with CoB, thus highlighting a critical contribution by TBI (Figure 1D). The result of immunosuppression-free allograft survival represents an incremental advance over data previously published by our group in which allograft survival was merely prolonged and required a short course of conventional immunosuppression (25). Similarly, in clinical BMT, standard conditioning or induction regimens generally include TBI to contribute to 
the eradication of the underlying disease and facilitate engraftment of donor hematopoieses (36), while promoting $\mathrm{T}$ cell apoptosis and resulting in fewer donor-reactive T cells 24 hours after induction $(37,38)$.

Additionally, however, one may postulate that the vascularized donor BM (DBM) compartment of a VCA, such as the orthotopic hind limb transplant used in this study, plays a critical role in achieving long-term graft survival. As recently reported, the BM niche is a dynamic environment rich in a variety of cells and has shown a significant survival benefit in small and large animal models $(25,32,39)$. In line with these findings, our results suggest that this VCA intrinsic feature - a viable BM component - allows for induction of stable multilineage mixed chimerism and long-term graft survival by TBI and CoB-based immunomodulation without the need for short- or long-term conventional maintenance immunosuppression. Based on our finding that donor-derived CD $11 \mathrm{c}^{+} \mathrm{DCs}$ were significantly more frequent in the thymus of animals tolerized with TBI + CoB (Supplemental Figure 6), our data further indicate that hematopoietic cells migrate from the donor marrow niche and achieve thymic engraftment. Furthermore, our data indicate that this specific treatment of BM-containing VCA and the resulting chimerism are establishing alloantigen-specific tolerance by intrathymic clonal deletion of donor-reactive $\mathrm{T}$ cell clones (Figure 6) $(14,24,33)$. Despite these findings, the definite role of the vBM niche in tolerance induction and the exact underlying mechanisms need to be further elucidated.

The central roles of the thymus and deletional tolerance mechanisms are well established in chimerism-based immunologic tolerance protocols. However, in addition, peripheral mechanisms dependent on Treg $\left(\mathrm{CD} 4^{+} \mathrm{Foxp}^{+}\right.$cells) were reported to play a critical role in the immune responses to solid organ transplants (27), as well as VCA (40). In studies of BMT with CoB, thymic-independent early deletion and anergy of alloreactive $\mathrm{CD}^{+} \mathrm{T}$ cells in the peripheral lymphoid organs have been demonstrated, and both activation-induced cell death and passive cell death initially contribute to peripheral $\mathrm{CD}^{+} \mathrm{T}$ effector cell deletion, highlighting a critical phenomenon for tolerance induction $(20,41)$. Recently, allograft biopsies of human hand transplants 3-6 years after transplantation stained positive for Foxp3 (42). These results further support the relevance of peripheral tolerance phenomena in maintenance of immune quiescence toward VCA. In our study, we found evidence of greater proportions of Tregs in the VCA (Figure 5C) and potent suppressive activities of Tregs (Supplemental Figure 4) that suggest a potential cocontribution of peripheral regulation and central deletional mechanisms (Figure 6 and Supplemental Figure 7) to tolerance maintenance in this murine VCA model. However, peripheral regulation by Tregs alone is not the main mechanism, as in vivo depletion did not affect tolerance induction (Supplemental Figure 6). Finally, in an attempt to determine the robustness of the established immune tolerance, we employed adoptive transfer of naive recipient-matched $\mathrm{T}$ cells into animals with a stable immune and graft status beyond POD 120 (Figure 7). The ensuing rejection of primary VCA (including secondary skin grafts) indicates a delicate balance of immune tolerance and alloimmunity within the host. Our data suggest this likely relies primarily on clonal deletion of donor-specific $\mathrm{T}$ cell clones during the establishment of mixed chimerism in the underlying treatment protocol. This is supported by findings in similar models of mixed chimerism in which long-term maintenance of donor-specific tolerance relies on intrathymic deletion following weakened peripheral regulatory mechanisms $(23,41,43)$. To understand the temporal role of the peripheral Treg response in tolerance induction and maintenance, further studies to assess functionality of infiltrating Tregs and mechanisms of balance between peripheral and central regulation by depleting Tregs will be required.

In conclusion, we were able to demonstrate long-term allograft survival and donor-specific immune tolerance in a fully mismatched murine model of VCA by using a combination of induction treatment with TBI and $\mathrm{CoB}$ without the need for conventional maintenance immunosuppression. We were further able to demonstrate a critical role of vBM featuring VCA in induction of donor-specific multilineage mixed chimerism. In our study, the induction of chimerism led to thymic engraftment and central deletion of alloreactive T effector cells and — combined with the presence of an intragraft Treg-dependent milieu — allow for immunosuppression-free allograft survival long-term.

\section{Methods}

Animals. Male 8- to 10-week-old Balb/C (H-2 $)$, C57BL/6 (B6; H-2 $)$, and FVB/N (H-2 $)$ mice were purchased from the Jackson Laboratory. Mice were housed in specific pathogen-free facilities at Johns Hopkins University.

Orthotopic hind limb transplantation and full-thickness skin transplantation. Balb/C hind limbs were transplanted into C57BL/6 recipients using a modified version of the technique described by Furtmüller et al. (15). Using the previously described 4-grade rejection scale, we defined Grade 3 (skin epidermolysis) as the endpoint in this study. 
Full-thickness trunk skin from Balb/C (donor-matched) or FVB/N (third-party) mice was transplanted onto the lateral thoracic wall of C57BL/6 recipients and secured with 6.0 Ethilon sutures (44). Skin graft survival was monitored daily. Rejection was defined as $70 \%$ necrosis of the skin graft.

Treatment protocols. Recipients were irradiated with a single, nonmyeloablative dose of 250 cGy using a Gammacell 40 one day before transplantation. Hamster anti-mouse CD154 mAb (MR1, BioXcell) and -CTLA4-Ig (Orencia [abatacep], Bristol-Myers Squibb) were administered on days 0, 2, 4, and 6 (500 $\mu \mathrm{g}$ / dose i.p.). The combination of anti-CD154 mAb and-CTLA4-Ig is referred to as CoB in this manuscript.

Tissue digestion and leukocytes isolation. Spleen, lymph nodes, thymus, and allografts were collected from transplanted mice at postoperative day (POD) 30, 50, 70, and 120. Cells from grafts were isolated using a modified technique of the methods described by Setoguchi et al. (45). Briefly, tissues were digested at $37^{\circ} \mathrm{C}$ via 3 consecutive 15 -minute incubations in PBS containing Collagenase IV (560U/mL; Worthington) DNAse I (275U/mL; Amresco), and Dispase II (0.4U/mL, Roche). Leukocytes were enriched using a $24 \%$ Histodenz-based (Sigma-Aldrich) gradient separation.

Flow cytometry analysis of mixed chimerism and donor-specific antibodies. The presence of donor hematopoiesis in VCA recipients was serially and quantitatively assessed by 4-color flow cytometry-based analysis (FACS Calibur flow cytometer [BD Biosciences] and FlowJo Software [Tree Star Inc.]) of donor-, recipient-, and cell lineage-specific cell surface markers on peripheral blood mononuclear cells (PBMCs). The percentage of donor cells circulating in host peripheral blood was calculated as described previously (46). FITC-conjugated $\mathrm{mAbs}$ directed against $\mathrm{H}-2^{\mathrm{d}}$ (clone SF1-1.1, BioLegend) and $\mathrm{H}-2^{\mathrm{b}}$ (clone AF6-885, BioLegend), PerCP-Cy5.5-conjugated anti-mouse CD3e (clone 145-2C11, BioLegend), PE-conjugated B220 (clone RA36B2, BioLegend), and Alexa Flour 647-conjugated CD11b (clone M1/70, BioLegend) were used.

The formation of donor-specific antibodies in VCA recipients was assessed by flow crossmatch-based analysis as previously described (47). Briefly, recipient serum (1:32 dilution) was incubated with Balb/c splenocytes $\left(1 \times 10^{6}\right)$ after blocking with FBS. APC-conjugated anti-CD3 mAb and FITC-conjugated anti-mouse IgG (BD Biosciences) was added to the samples. The DSA level of each sample was calculated as MFI.

Flow cytometry analysis of TCR VB TCR families. Specific PE-conjugated monoclonal antibodies against V $\beta 5.1 / 2$ (clone MR9-4, BD Biosciences), V $\beta 11$ (clone RR3-15, BD Biosciences), or V $\beta 8.1 / 2$ (clone F23.1, BD Biosciences) were used to stain peripheral blood lymphocytes at multiple time points for evidence of central deletion of transgenic V $\beta$ TCR repertoires. Additionally, samples were stained with PerCP-Cy5.5conjugated anti-CD3e, Pacific Blue-conjugated anti-CD4 (clone RM4-5, Thermo Fisher Scientific), and APC-conjugated anti-CD8a (clone 53-6.7, Thermo Fisher Scientific). The percentage of $\mathrm{CD}^{+} \mathrm{CD}^{+}$cells expressing each $\mathrm{V} \beta$ was determined.

Flow cytometry analysis of intracellular cytokine and transcription factor expression. The following fluorochrome-tagged antibodies were purchased from BD Pharmingen, Thermo Fisher Scientific, and BioLegend: CD4-PB, CD8-FITC, IFN- $\gamma$-PE/Cy7 (clone XMG1.2, BD Biosciences), IL-2-PE (clone JES6-5H4, Thermo Fisher Scientific), IL-17-PerCP-Cy5.5 (clone eBo17B7, Thermo Fisher Scientific), IL-10-APC (clone JES5-16E3, Thermo Fisher Scientific), T-bet-PE-Cy7 (clone 4B10, Thermo Fisher Scientific), GATA3-APC (clone L50-823, BD Biosciences), RoR- $\gamma$ t-PE (clone Q31-378, BD Biosciences), and FoxP3PerCP-Cy5.5 (clone FJK-16s, Thermo Fisher Scientific). Splenocytes and lymph node cells were stimulated in vitro for intracellular cytokines with PMA/ionomycin and protein transport cocktail (Brefeldin/Monensin, eBioscience) for 4.5 hours. Intracellular cytokine staining was performed according to the manufacturer's protocol. Briefly, cells were washed, stained for surface markers, fixed with BD Cytofix/Cytoperm, permeabilized, and incubated with antibodies against intracellular markers for 40 minutes on ice. Flow cytometry-based data acquisition was performed on an LSRII (BD Biosciences), and data were analyzed using FlowJo software.

$T$ cell and BM-derived DC isolations and in vitro MLR. T cell isolation was performed via negative selection of splenocytes and lymphocytes of recipient mice using magnetic bead sorting for in vitro MLR assays and for in vivo testing of peripheral regulatory mechanism via naive donor-type T cell injection (i.e., $20 \times 10^{6} \mathrm{C} 57 \mathrm{BL} / 6$ mouse $\mathrm{T}$ cells via tail vein). Cells were incubated using purified rat anti-mouse B220, Gr-1, TER-119, I-A/ I-E, CD11b, and CD16/32 and anti-rat IgG Dynabeads (Invitrogen), leading to a CD4 ${ }^{+} \mathrm{T}$ cell-enriched population following magnetic selection. CFSE (Thermo Fisher Scientific) labeling of T cells was performed according to the manufacture's protocol.

As stimulator cells in MLR assays, DCs were generated from both donor-matched (Balb/C) and third-party derived (FVB/N) BM after culture for 7 days with IL-4 and GM-CSF in a $37^{\circ} \mathrm{C}$ incubator (48). 
Twenty-four hours prior to MLR plating, DCs were activated with $200 \mathrm{ng} / \mathrm{mL}$ of LPS. CD11c ${ }^{+}$DCs were then isolated using positive magnetic sorting according to the manufacturer's protocol (Mouse CD11c Positive Selection Kit, Stemcell Technologies). CFSE-labeled T cells and DCs were cultured for 4 days in $37^{\circ} \mathrm{C}$, collected, and stained with Fixable Viability Dye (Thermo Fisher Scientific) according to the manufacturer's protocols. Samples were stained for CD4 and CD8, and data were acquired on an LSRII. Data were analyzed using FlowJo software (Tree Star Inc.), and results were normalized to the proliferation of naive cells within each individual experiment.

Suppression assay with Tregs. CD4 ${ }^{+} \mathrm{T}$ cell isolation was performed via negative selection of splenocytes and lymphocytes of mice using magnetic bead sorting. Cells were incubated using purified rat anti-mouse B220, Gr-1, TER-119, I-A/I-E, CD11b, CD8, and CD16/32 and anti-rat IgG Dynabeads (Invitrogen), leading to a $\mathrm{CD}^{+} \mathrm{T}$ cell-enriched population following magnetic selection. $\mathrm{CD} 25^{+}$selection was followed using magnetic bead sorting (Stemcell Technologies). Overall purity was over $90 \%$ in Foxp3 $3^{+}$cell selection. Carboxyfluorescein diacetate succinimidyl ester (CFSE, Thermo Fisher Scientific) labeling of conventional T cells $\left(\mathrm{CD}^{+} \mathrm{CD} 25^{-}\right)$was performed according to the manufacture's protocol. Autologous BM-derived DCs and anti-CD3 antibodies were used for stimulation. CFSE-labeled conventional T cells, DCs, and anti-CD3 in the presence or absence of various ratios of Treg were cultured for 3 days in $37^{\circ} \mathrm{C}$, collected, and stained with Fixable Viability Dye (Thermo Fisher Scientific) according to the manufacturer's protocol. Samples were stained for CD4, and data were acquired on an LSRII. Data were analyzed using FlowJo software.

Histological analysis. Fresh tissue was fixed in 10\% formalin for at least 24 hours and subsequently processed and embedded in paraffin. Tissue sections $(5 \mu \mathrm{m}$-thick) were cut on a microtome and stained with H\&E. Histological specimens were reviewed by a mouse pathologist blinded to the treatment regimens.

Statistics. Allograft survival was calculated according to the Kaplan-Meier product limit method and compared between groups using a log-rank test. Statistical analysis of in vitro data pertaining to donor-specific chimerism, cell proliferation, and the frequency of $\mathrm{T}$ cells, transcription factors ILs was determined with a 2-tailed Student's $t$ test for comparison of means with unequal variances. A $P$ value of less than 0.05 was considered statistically significant.

Study approval. All experiments were approved by the IACUC of Johns Hopkins University (IACUC no. M019M352) and conducted according to NIH guidelines.

\section{Author contributions}

BCO, GJF, MLF, YG, FM, and JK performed surgical procedures, conducted in vitro experiments, cared for experimental animals, interpreted data, and prepared manuscript. SS, DSC, WPAL, and GR interpreted data and prepared the manuscript. GB designed experiments, interpreted data, and prepared manuscript.

\section{Acknowledgments}

The authors thank Richa Kalsi (Department of Surgery, University of Maryland, Baltimore, Maryland, USA) for her help with the graphic abstract for this manuscript. We would like to acknowledge internal support from the Department of Plastic and Reconstructive Surgery, Johns Hopkins University School of Medicine.

Address correspondence to: Gerald Brandacher, Ross Research Building/Room 749D, 720 Rutland Avenue, Baltimore, Maryland 21205, USA. Phone: 1.443.287.6679; Email: brandacher@jhmi.edu.

1. Shores JT, Brandacher G, Lee WP. Hand and upper extremity transplantation: an update of outcomes in the worldwide experience. Plast Reconstr Surg. 2015;135(2):351e-360e.

2. Guillot C, et al. Tolerance to cardiac allografts via local and systemic mechanisms after adenovirus-mediated CTLA4Ig expression. J Immunol. 2000;164(10):5258-5268.

3. Guillot C, et al. Active suppression of allogeneic proliferative responses by dendritic cells after induction of long-term allograft survival by CTLA4Ig. Blood. 2003;101(8):3325-3333.

4. Kinnear G, Jones ND, Wood KJ. Costimulation blockade: current perspectives and implications for therapy. Transplantation. 2013;95(4):527-535

5. Riella LV, Sayegh MH. T-cell co-stimulatory blockade in transplantation: two steps forward one step back! Expert Opin Biol Ther 2013;13(11):1557-1568.

6. Larsen CP, Knechtle SJ, Adams A, Pearson T, Kirk AD. A new look at blockade of T-cell costimulation: a therapeutic strategy for long-term maintenance immunosuppression. Am J Transplant. 2006;6(5 Pt 1):876-883.

7. Gilson CR, et al. Anti-CD40 monoclonal antibody synergizes with CTLA4-Ig in promoting long-term graft survival in murine 
models of transplantation. J Immunol. 2009;183(3):1625-1635.

8. Trambley J, et al. Asialo GM1(+) CD8(+) T cells play a critical role in costimulation blockade-resistant allograft rejection. J Clin Invest. 1999;104(12):1715-1722.

9. Bluestone JA, St Clair EW, Turka LA. CTLA4Ig: bridging the basic immunology with clinical application. Immunity. 2006;24(3):233-238.

10. Kirk AD, et al. Treatment with humanized monoclonal antibody against CD154 prevents acute renal allograft rejection in nonhuman primates. Nat Med. 1999;5(6):686-693.

11. Larsen CP, et al. Long-term acceptance of skin and cardiac allografts after blocking CD40 and CD28 pathways. Nature. 1996;381(6581):434-438.

12. Page A, et al. CD40 blockade combines with CTLA4Ig and sirolimus to produce mixed chimerism in an MHC-defined rhesus macaque transplant model. Am J Transplant. 2012;12(1):115-125.

13. Taylor PA, Lees CJ, Waldmann H, Noelle RJ, Blazar BR. Requirements for the promotion of allogeneic engraftment by antiCD154 (anti-CD40L) monoclonal antibody under nonmyeloablative conditions. Blood. 2001;98(2):467-474.

14. Wekerle T, et al. Allogeneic bone marrow transplantation with co-stimulatory blockade induces macrochimerism and tolerance without cytoreductive host treatment. Nat Med. 2000;6(4):464-469.

15. Furtmüller GJ, et al. Orthotopic Hind Limb Transplantation in the Mouse. J Vis Exp. 2016;(108):53483.

16. Williams MA, et al. Genetic characterization of strain differences in the ability to mediate CD40/CD28-independent rejection of skin allografts. J Immunol. 2000;165(12):6849-6857.

17. Wekerle T, Kurtz J, Bigenzahn S, Takeuchi Y, Sykes M. Mechanisms of transplant tolerance induction using costimulatory blockade. Curr Opin Immunol. 2002;14(5):592-600.

18. Joffre $\mathrm{O}$, et al. Prevention of acute and chronic allograft rejection with CD4+CD25+Foxp3+ regulatory T lymphocytes. Nat Med. 2008;14(1):88-92.

19. Tsang JY, et al. Conferring indirect allospecificity on CD4+CD25+ Tregs by TCR gene transfer favors transplantation tolerance in mice. J Clin Invest. 2008;118(11):3619-3628.

20. Wekerle T, et al. Extrathymic T cell deletion and allogeneic stem cell engraftment induced with costimulatory blockade is followed by central T cell tolerance. J Exp Med. 1998;187(12):2037-2044.

21. Thomas-Vaslin V, Salaün J, Coltey M, Vaigot P, Fucs R. Kinetics and repertoire selection of T cells derived from the early waves of fetal thymus colonization after thymus grafting in allogeneic nude recipients. Scand J Immunol. 1997;45(5):482-486.

22. Adams AB, Ford ML, Larsen CP. Costimulation Blockade in Autoimmunity and Transplantation: The CD28 Pathway. J Immunol. 2016;197(6):2045-2050.

23. Kurtz J, Wekerle T, Sykes M. Tolerance in mixed chimerism - a role for regulatory cells? Trends Immunol. 2004;25(10):518-523.

24. Wekerle T, Sykes M. Mixed chimerism and transplantation tolerance. Annu Rev Med. 2001;52:6

25. Lin CH, et al. Combined Anti-CD154/CTLA4Ig Costimulation Blockade-Based Therapy Induces Donor-Specific Tolerance to Vascularized Osteomyocutaneous Allografts. Am J Transplant. 2016;16(7):2030-2041.

26. Lin $\mathrm{H}$, et al. Long-term acceptance of major histocompatibility complex mismatched cardiac allografts induced by CTLA4Ig plus donor-specific transfusion. J Exp Med. 1993;178(5):1801-1806.

27. Sucher R, et al. IDO and regulatory T cell support are critical for cytotoxic T lymphocyte-associated Ag-4 Ig-mediated longterm solid organ allograft survival. J Immunol. 2012;188(1):37-46.

28. Kirk AD, et al. CTLA4-Ig and anti-CD40 ligand prevent renal allograft rejection in primates. Proc Natl Acad Sci USA . 1997;94(16):8789-8794.

29. Larsen CP, et al. Rational development of LEA29Y (belatacept), a high-affinity variant of CTLA4-Ig with potent immunosuppressive properties. Am J Transplant. 2005;5(3):443-453.

30. Shirasugi N, et al. Prevention of chronic rejection in murine cardiac allografts: a comparison of chimerism- and nonchimerism-inducing costimulation blockade-based tolerance induction regimens. J Immunol. 2002;169(5):2677-2684.

31. Siemionow M, Klimczak A, Unal S, Agaoglu G, Carnevale K. Hematopoietic stem cell engraftment and seeding permits multi-lymphoid chimerism in vascularized bone marrow transplants. Am J Transplant. 2008;8(6):1163-1176.

32. Barth RN, et al. Vascularized bone marrow-based immunosuppression inhibits rejection of vascularized composite allografts in nonhuman primates. Am J Transplant. 2011;11(7):1407-1416.

33. Adams AB, et al. Costimulation blockade, busulfan, and bone marrow promote titratable macrochimerism, induce transplantation tolerance, and correct genetic hemoglobinopathies with minimal myelosuppression. J Immunol. 2001;167(2):1103-1111.

34. Zhang T, Pierson RN, Azimzadeh AM. Update on CD40 and CD154 blockade in transplant models. Immunotherapy. 2015;7(8):899-911.

35. Nanji SA, et al. Costimulation blockade of both inducible costimulator and CD40 ligand induces dominant tolerance to islet allografts and prevents spontaneous autoimmune diabetes in the NOD mouse. Diabetes. 2006;55(1):27-33.

36. Sharabi Y, Sachs DH. Mixed chimerism and permanent specific transplantation tolerance induced by a nonlethal preparative regimen. J Exp Med. 1989;169(2):493-502.

37. Fujimori Y, Saheki K, Itoi H, Okamamoto T, Kakishita E. Increased expression of Fas (APO-1, CD95) on CD4+ and CD8+ T lymphocytes during total body irradiation. Acta Haematol. 2000;104(4):193-196.

38. Kurtz J, Ito H, Wekerle T, Shaffer J, Sykes M. Mechanisms involved in the establishment of tolerance through costimulatory blockade and BMT: lack of requirement for CD40L-mediated signaling for tolerance or deletion of donor-reactive CD4+ cells Am J Transplant. 2001;1(4):339-349.

39. Siemionow $\mathrm{M}$, et al. The reversed paradigm of chimerism induction: Donor conditioning with recipient-derived bone marrow cells as a novel approach for tolerance induction in vascularized composite allotransplantation. Microsurgery. 2016;36(8):676-683.

40. Lin $\mathrm{CH}$, et al. Vascularized osteomyocutaneous allografts are permissive to tolerance by induction-based immunomodulatory therapy. Am J Transplant. 2013;13(8):2161-2168.

41. Kurtz J, Shaffer J, Lie A, Anosova N, Benichou G, Sykes M. Mechanisms of early peripheral CD4 T-cell tolerance induction by anti-CD154 monoclonal antibody and allogeneic bone marrow transplantation: evidence for anergy and deletion but not regulatory cells. Blood. 2004;103(11):4336-4343. 
42. Eljaafari A, et al. Isolation of regulatory $\mathrm{T}$ cells in the skin of a human hand-allograft, up to six years posttransplantation. Transplantation. 2006;82(12):1764-1768.

43. Ito H, Kurtz J, Shaffer J, Sykes M. CD4 T cell-mediated alloresistance to fully MHC-mismatched allogeneic bone marrow engraftment is dependent on CD40-CD40 ligand interactions, and lasting T cell tolerance is induced by bone marrow transplantation with initial blockade of this pathway. J Immunol. 2001;166(5):2970-2981.

44. Cheng CH, et al. Murine Full-thickness Skin Transplantation. J Vis Exp. 2017;(119):55105.

45. Setoguchi K, et al. LFA-1 antagonism inhibits early infiltration of endogenous memory CD8 T cells into cardiac allografts and donor-reactive T cell priming. Am J Transplant. 2011;11(5):923-935.

46. Pree I, Wekerle T. Inducing mixed chimerism and transplantation tolerance through allogeneic bone marrow transplantation with costimulation blockade. Methods Mol Biol. 2007;380:391-403.

47. Oh B, Yoon J, Farris A, Kirk A, Knechtle S, Kwun J. Rapamycin Interferes With Postdepletion Regulatory T Cell Homeostasis and Enhances DSA Formation Corrected by CTLA4-Ig. Am J Transplant. 2016;16(9):2612-2623.

48. Raimondi G, et al. Mammalian target of rapamycin inhibition and alloantigen-specific regulatory $\mathrm{T}$ cells synergize to promote long-term graft survival in immunocompetent recipients. J Immunol. 2010;184(2):624-636. 